\title{
Thermal resonance in signal transmission
}

\author{
Ramon Reigada, ${ }^{*}$ Antonio Sarmiento, ${ }^{\dagger}$ and Katja Lindenberg \\ Department of Chemistry and Biochemistry 0340, University of California, San Diego, La Jolla, California $92093-0340$
}

(Received 7 June 2000; revised manuscript received 18 January 2001; published 21 May 2001)

\begin{abstract}
We use temperature tuning to control signal propagation in simple one-dimensional arrays of masses connected by hard anharmonic springs and with no local potentials. In our numerical model a sustained signal is applied at one site of a chain immersed in a thermal environment and the signal-to-noise ratio is measured at each oscillator. We show that raising the temperature can lead to enhanced signal propagation along the chain, resulting in thermal resonance effects akin to the resonance observed in arrays of bistable systems.
\end{abstract}

DOI: 10.1103/PhysRevE.63.066113

PACS number(s): 05.40.Ca, 02.50.Ey, 05.45.Xt

\section{INTRODUCTION}

In the past few years it has become abundantly clear that the presence of noise in nonlinear systems may lead to an enhancement of a number of often desirable features such as energy localization and mobility and the detection and propagation of weak signals. The interplay of stochasticity and nonlinearity that amplifies the system response is a cooperative phenomenon whose detailed nature depends on the particular structure of the system and the forces acting upon it $[1,2]$. One manifestation of the interplay is found in the phenomenon called stochastic resonance, which has been invoked in a wide range of physical [3-5], chemical [6-9], geological $[1,10]$, and biological $[8,11]$ systems. Recent literature, including our own work [12-14], has focused on spatially extended systems [15] including noise-enhanced propagation in coupled arrays of bistable units $[3,16]$, excitable media [7,8,17], reaction-diffusion systems [18], and dynamics and signal propagation in cardiac tissue $[19,20]$. It has been repeatedly noted that discrete extended systems pose particular mathematical challenges that have barely been explored in spite of the fact that many physical systems are intrinsically discrete [21-25].

The ubiquitous picture of stochastic resonance involves a particle moving in a double-well potential subject to a weak external signal that periodically changes the potential by alternately raising and lowering the wells [2]. The signal is "weak" if the periodic force is too small to cause the particle to scale the barrier between the wells. Nevertheless, an appropriate random force is sufficient to cause the particle to cross over the barrier even in the absence of a deterministic signal. In the simultaneous presence of a weak signal and a sufficiently weak noise, the transitions over the barrier occur rarely and at a rate determined by the noise intensity. These transitions are slow compared to the frequency of the deterministic signal; the transition rate then carries little information about the signal. At the other extreme, when the noise is strong it induces rapid transitions that are again essentially uninfluenced by the frequency of the signal. At an optimal

\footnotetext{
* Permanent address: Departament de Quimica-Fisica, Universitat de Barcelona, Avda. Diagonal 647, 08028 Barcelona, Spain.

†Permanent address: Instituto de Matematicas, UNAM, Avenida Universitaria s/n, Chamilpa, Morelos 62200, Mexico.
}

noise intensity, however, the mean first passage time associated with the noise and the frequency of the signal are in synchrony (stochastic resonance), and the passage from one well to the other carries maximal information about the signal frequency.

A less ubiquitous but nonetheless important occurrence of stochastic resonance (that has been called "nonconventional" by its discoverers) arises for particles moving in nonlinear monostable potentials [26,27]. It is argued that stochastic resonance can be expected to occur in any single-well underdamped system for which the spectral density of the fluctuations of the system in the absence of a periodic signal exhibits a well-resolved narrow peak that grows faster than quadratically with temperature. The effect is confirmed via analog simulations of a single-well Duffing oscillator [26,27] and of a superconducting quantum interference device loop [28]. More recently, stochastic resonance at higher harmonics in monostable systems was ascertained for an overdamped system when the nonlinearity is not concentrated at the equilibrium position [29].

Recent developments in the field have generalized these ideas to linearly coupled arrays of bistable oscillators $[3,4,16]$. A signal with the help of the noise in these arrays can cause a "phase jump." If the noise is sufficiently weak, the phase jump travels in the form of a moving kink (strong noise causes random phase jumps that make it difficult to separately identify a phase jump associated with the signal). The creation or destruction of a phase kink is an activated process, i.e., the signal and/or noise must be sufficiently strong to cause a transition from one well of one of the bistable potentials to the other. The presence of such kinks is associated with an "energy gap:" it takes a finite amount of energy to destroy a kink. The language used in this description was originally borrowed from the kink soliton context.

Reported instances of stochastic enhancement and stochastic resonance in extended arrays involve coupled (overdamped) bistable units. Herein we show that enhanced propagation can be achieved through thermal tuning of even simpler discrete arrays of masses connected by monostable anharmonic springs (with no local potentials). The signal is identified with an amplitude that exceeds (by a predetermined amount) that due to the thermal background. Here there is no activation process and no energy gap, and the signal can simply disperse or dissipate. The language 

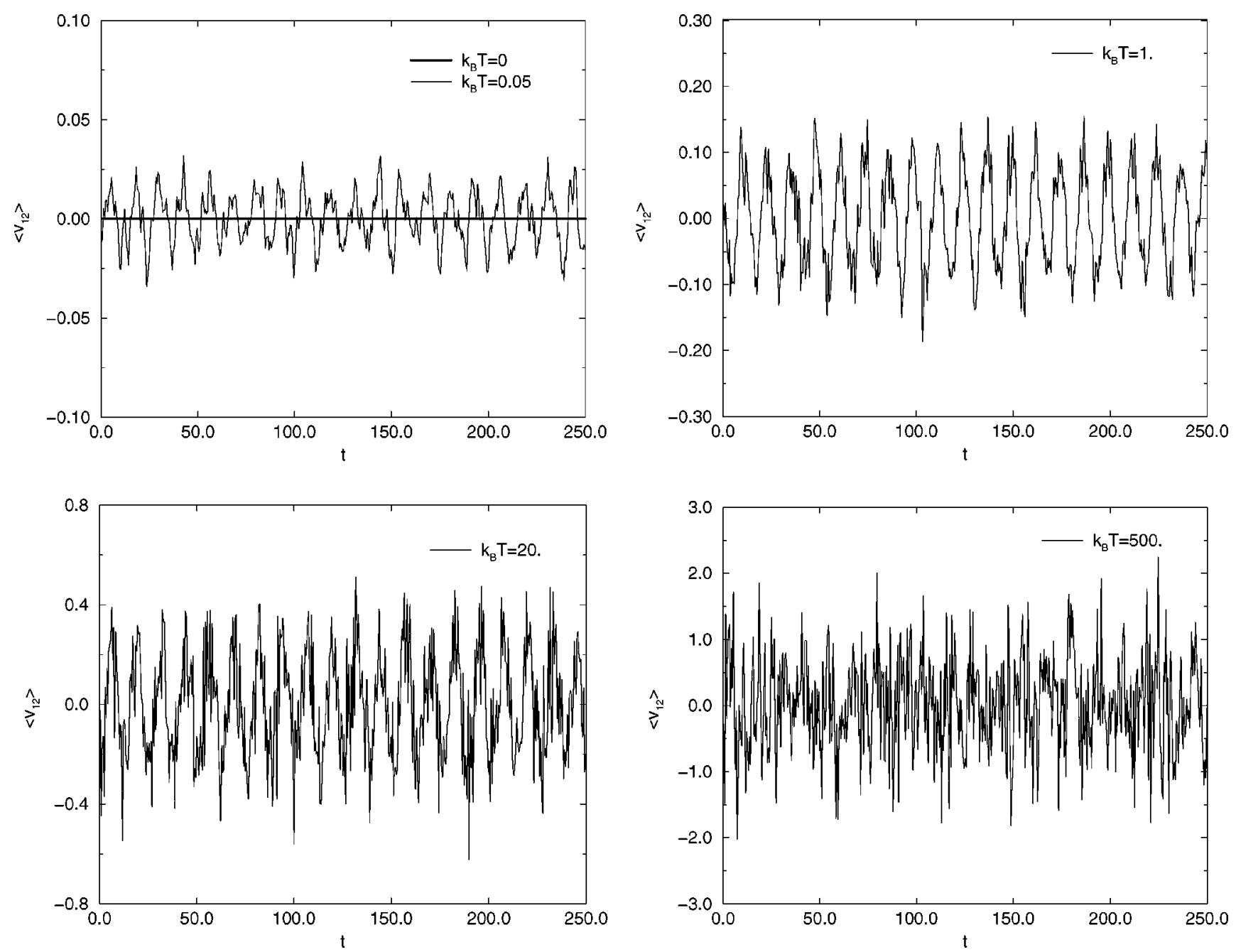

FIG. 1. Mean velocity $\langle v\rangle$ of the 12th site of a hard anharmonic chain with $k^{\prime}=3, \gamma=0.5, \omega_{0}=0.5$, and $A=0.5$, for different values of the temperature. Enhancement of signal propagation is observed with increasing temperature (1st and 2nd panels), but the signal is destroyed as the temperature further increases (3rd and 4 th panels). Note the different vertical scales.

appropriate to this case is akin to that originally associated with envelope solitons. We focus on the propagation distance and amplitude along the chain of a signal continuously applied at one site of a one-dimensional array, and compare results for harmonic and hard anharmonic chains. In particular, we show that when the anharmonic chain is immersed in a thermal bath, it is possible to maximize the distance of propagation and the amplitude of the signal at a given site by tuning the temperature to particular optimal values. We call this phenomenon thermal resonance. Our systems are in general not overdamped and thus include inertial contributions to the motions of the masses. Noise and damping represent a realistic thermal environment with a tunable temperature and dissipation that obey an appropriate fluctuation-dissipation relation. We have found and reported elsewhere [13] that the propagation of an energy pulse in a hard anharmonic array can be enhanced by immersion in a thermal bath, and that hard anharmonicity in the springs causes a tight and persistent packing of the energy. Those results suggest the possibility of a thermal resonance in the transport of a sustained external signal in these simple arrays.
In Sec. II we present our model and some details of the numerical integration of the equations of motion. Our characterization of a thermal resonance is presented in Sec. III, and our main results are shown in Sec. IV. In Sec. V we discuss the dependence of our results on different parameter models. Section VI contains our summary and conclusions.

\section{THE MODEL AND NUMERICAL PROCEDURE}

Our model consists of a one-dimensional chain of $N$ unitmass sites, each connected to its nearest neighbors by either harmonic (quadratic) or hard anharmonic (quartic) springs. Accordingly, the Hamiltonian of the array is

$$
H=\sum_{n=0}^{N-1}\left[\frac{p_{n}^{2}}{2}+\frac{k}{2}\left(x_{n}-x_{n-1}\right)^{2}+\frac{k^{\prime}}{4}\left(x_{n}-x_{n-1}\right)^{4}\right],
$$

where $k$ and $k^{\prime}$ are the harmonic and anharmonic coupling constants respectively. Thermalization of the chains is achieved through a Langevin prescription for coupling the system to a heat bath. The stochastic equations of motion for 

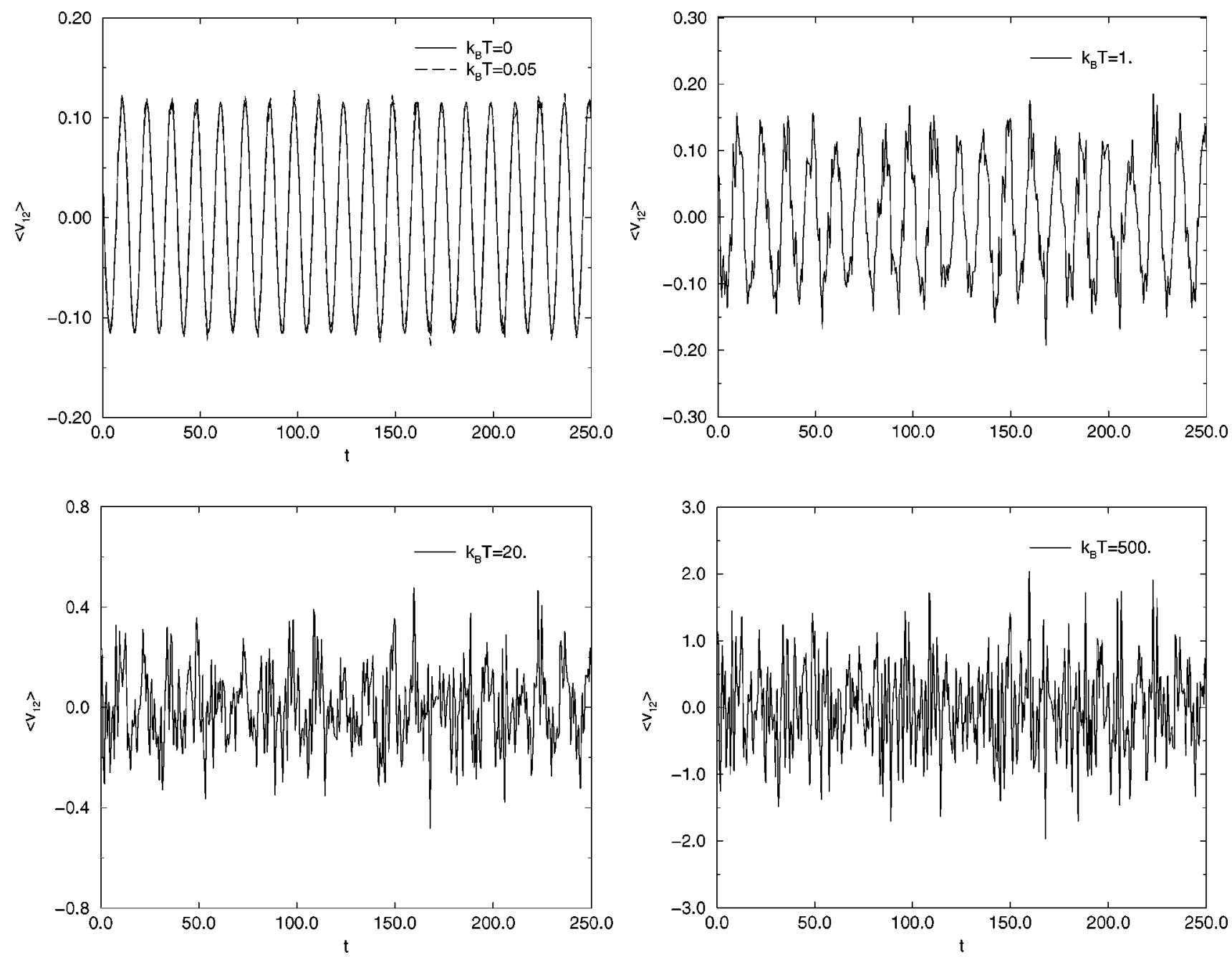

FIG. 2. Mean velocity $\langle v\rangle$ of the 12 th site of the harmonic chain with $k=3, \gamma=0.5, \omega_{0}=0.5$, and $A=0.5$, for different values of the temperature. Signal degradation with increasing temperature is clearly observed. Note the different vertical scales.

sites $n=1, \ldots, N-1$ are obtained from the Hamiltonian augmented by the usual Langevin forces,

$$
\begin{aligned}
\ddot{x}_{n}= & k\left(x_{n-1}-x_{n}\right)-k\left(x_{n}-x_{n+1}\right)+k^{\prime}\left(x_{n-1}-x_{n}\right)^{3} \\
& -k^{\prime}\left(x_{n}-x_{n+1}\right)^{3}-\gamma \dot{x}_{n}+f_{n}(t),
\end{aligned}
$$

where a dot represents a derivative with respect to time. The $f_{n}(t)$ are zero-centered, Gaussian, $\delta$-correlated fluctuations that satisfy the fluctuation-dissipation relation at temperature $T$,

$$
\left\langle f_{n}(t) f_{n^{\prime}}\left(t^{\prime}\right)\right\rangle=2 \gamma k_{B} T \delta_{n, n^{\prime}} \delta\left(t-t^{\prime}\right)
$$

( $k_{B}$ is Boltzmann's constant). We impose periodic boundary conditions, so that $x_{N} \equiv x_{0}$. A sustained signal is applied to the site $n=0$ that determines its velocity at all times,

$$
\dot{x}_{0}=A \sin \left(\omega_{0} t\right) \text {. }
$$

The positions and momenta of all the other sites are otherwise "free" and determined by the equations of motion. We study the propagation of this signal along the chain as a function of the temperature. This particular way of applying a signal is of course not unique (e.g., one might apply an oscillatory force instead), but we have ascertained that the results are insensitive to the detailed choice.

An analytic solution of this problem is not available for an anharmonic chain, so we must rely on numerical integration, which is performed using the second order Heun's method (equivalent to a second order Runge-Kutta integration) [30,31]. The time step is determined by the period of oscillation of the velocity of the first site $\tau=2 \pi / \omega_{0}$ through the relation $\Delta t=\tau / 2^{12}$. For each simulation, the system is initially allowed to relax to thermal equilibrium. For all the simulations presented, this is achieved in less than 20 units of the dimensionless time. Typically, after a transient that is longer the farther the site is from site 0 (and thus a measure of the velocity of propagation), each site settles into stationary behavior that is a mixture of thermal motion and response to the signal. Sites that are far from the signal never exhibit this transient (thus indicating a finite distance of propagation) and simply continue their thermal motion. At any given site that is reached by the signal, one can observe the amplitude of the motion associated with the signal over and above the thermal motion. 
Our interest here lies in demonstrating resonances in the dependence on temperature of the propagation distance and velocity and of the response amplitudes once stationarity has been achieved (in all our simulations, each site has settled into its long-time behavior after 100 first-site oscillations). Possible energy return effects around the periodic chain are prevented by making the chain sufficiently long and/or sufficiently increasing the dissipation parameter of distant sites. Our chains typically consist of 70 sites with a large dissipation at sites 27-32 (these numbers can easily be varied). Our "measurements" are then taken over 80 oscillation periods and nonzero-temperature results are averaged over 500 realizations. All of these choices (equilibration time before applying the signal, integration time step, transients, length of chain, and number of realizations) have been carefully tested.

\section{CHARACTERIZATION OF THERMAL RESONANCE}

We must choose a sensible response variable to characterize the behavior of our array. When stochastic resonance is studied in arrays of bistable potentials, the system response is usually analyzed in terms of a crossover time series that characterizes the transitions of each bistable element from one well to the other. In excitable media a reasonable response variable involves firing times of the individual elements. In our system the most convenient choice is the velocity of each site because it corresponds directly to the applied signal, and because the time average of the velocity in the stationary state vanishes at any temperature.

Figure 1 is a dramatic but typical demonstration of a realization of thermal resonance. It shows the mean velocity $\langle v\rangle$ (averaged over realizations) as a function of time for the 12 th site of a hard anharmonic chain at different temperatures. The first panel shows the results for zero temperature and for a very low temperature; the temperature increases in subsequent panels. At zero temperature the 12 th site hardly moves because the signal has been dissipated to the bath before reaching this site (in the corresponding harmonic chain the signal reaches the 12 th site quite vigorously at zero temperature, a confirmation of the fact that a given dissipation is much more effective in a hard anharmonic potential than in a harmonic one $[12,13,32]-$ see Fig. 2). A very small temperature increase (still first panel) causes a large enhancement of the signal, which clearly now reaches the site. This is apparent in the oscillatory behavior of the velocity over and above the noisy background. The temperature in the second panel is close to its optimal value, that is, the value that most enhances the signal at this particular site relative to the thermal background. Hence the motion of the 12th site at this temperature is mostly driven by the periodic forcing of the first site. A further increase in the temperature (third panel) causes the average velocity to become increasingly noisy because ever larger fluctuations dominate the dynamics. Finally, at a sufficiently high temperature (fourth panel), the signal is essentially buried in the fluctuations, and the motion is simply that imposed by the thermal bath. In contrast, in a harmonic array $(k=3)$ the signal at any site simply degrades with increasing temperature. This is illustrated in Fig. 2.

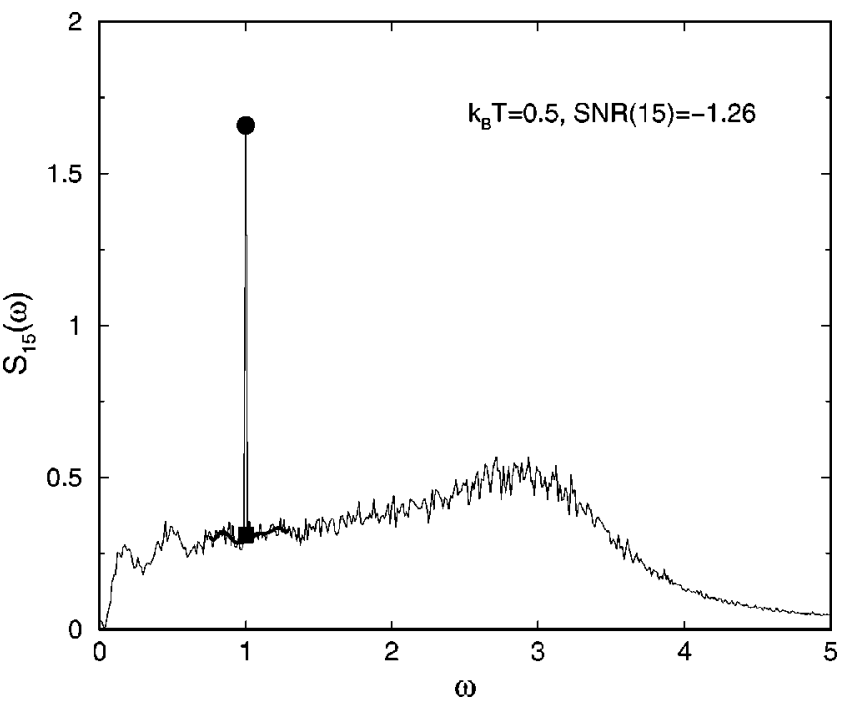

FIG. 3. Power spectral density at the 15 th site of the hard anharmonic chain with $k^{\prime}=5, \gamma=0.2, \omega_{0}=1.0$ and $A=0.5$ (we call this our "standard case" in the text) at temperature $k_{B} T=0.5$. The wide solid line shows the polynomial fitting around $\omega_{0}$. The circle indicates the value of $S_{15}\left(\omega_{0}\right)$ and the square that of $S_{15 \text {, noise }}\left(\omega_{0}\right)$.

To provide a quantitative measure of the thermal resonance, we define the power spectral density $S_{j}(\omega)$ at each site $j$ as

$$
S_{j}(\omega)=\int_{-\infty}^{+\infty} e^{-i \omega \tau}\left\langle v_{j}(t) v_{j}(t+\tau)\right\rangle d \tau
$$

where the brackets denote an ensemble average over realizations and an average over time. In addition to the thermal fluctuations, this function contains the spectral information about that part of the signal that has reached site $j$. An example of a portion of the spectrum for the case that we will call our "standard case" $\left(k^{\prime}=5, \quad \gamma=0.2, \omega_{0}=1.0, \quad A\right.$ $=0.5$ ) is shown in Fig. 3. The signal extraction from background noise that characterizes stochastic resonance is traditionally performed via a signal-to-noise ratio (SNR) [2]:

$$
\mathcal{R}(j) \equiv \log _{10}\left\{\frac{[\text { signal power }(j)] \times \Delta \omega}{[\text { thermal power }(j)]}\right\},
$$

where the signal power is the value $S_{j}\left(\omega_{0}\right)-S_{j, \text { noise }}\left(\omega_{0}\right)$, the thermal power $S_{j, \text { noise }}\left(\omega_{0}\right)$ is estimated by performing a fourth-order polynomial fit to $S_{j}(\omega)$ around-but not including - the forcing frequency $\omega_{0}$, and $\Delta \omega$ denotes the frequency integration step and is equal to 0.0125 (the inverse of the 80 oscillation periods used as our measurement time) throughout this paper. This definition of the SNR is not unique, but our results are robust with respect to variations in this definition.

\section{THERMAL RESONANCES}

We first present SNR results for the harmonic chain, so as to clarify later the ways in which the anharmonic chain behaves differently. The analytic calculations associated with 

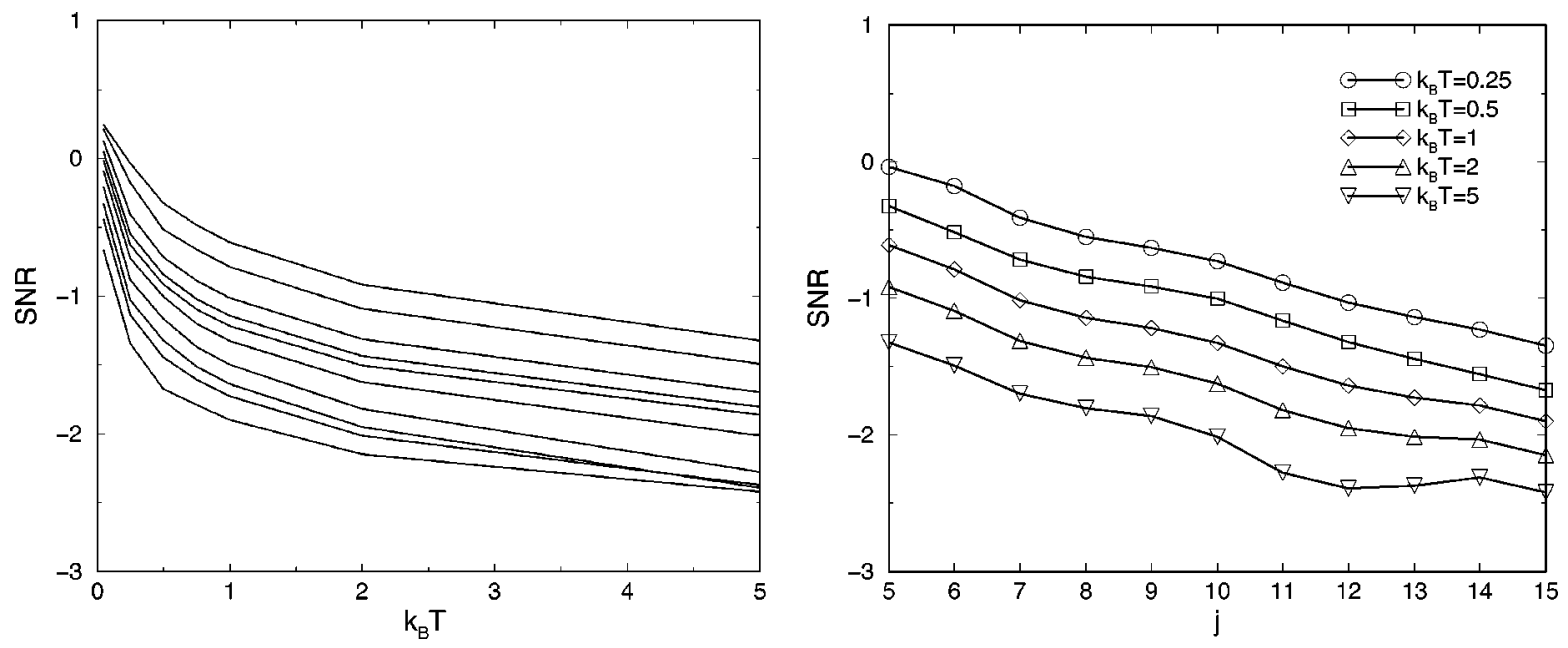

FIG. 4. First panel: Typical SNR curves as a function of temperature for different sites along a harmonic chain. The SNR for each site decreases monotonically with temperature. This particular example shows sites from the 5th to the 14 th (top to bottom) with $k=3$, $\gamma$ $=0.5, \omega_{0}=1.0$, and $A=0.5$. Second panel: SNR as a function of site for different temperatures.

the harmonic chain are presented in the Appendix. These results serve as a test for our numerical simulations. The first panel in Fig. 4 shows SNR curves as a function of temperature for different sites, and the second panel shows the same results as a function of distance from the forced site for different temperatures. The results are exactly as shown in the Appendix and as one would expect: the SNR decreases monotonically with increasing temperature and with increasing distance from the applied signal. Note that $\mathcal{R}(j)$ decreases with increasing temperature because the numerator in Eq. (6) is essentially independent of temperature while the denominator increases (see the Appendix). A point to note is that the decay of $\mathcal{R}(j)$ with $j$ at a given temperature provides a measure of the shape of the stationary front of the signal at that temperature.

We now turn to the anharmonic chain. The first panel in Fig. 5 shows SNR curves as a function of temperature for different sites. The thermal resonance is identified with the $j$-dependent maximum of $\mathcal{R}(j)$ as a function of temperature.

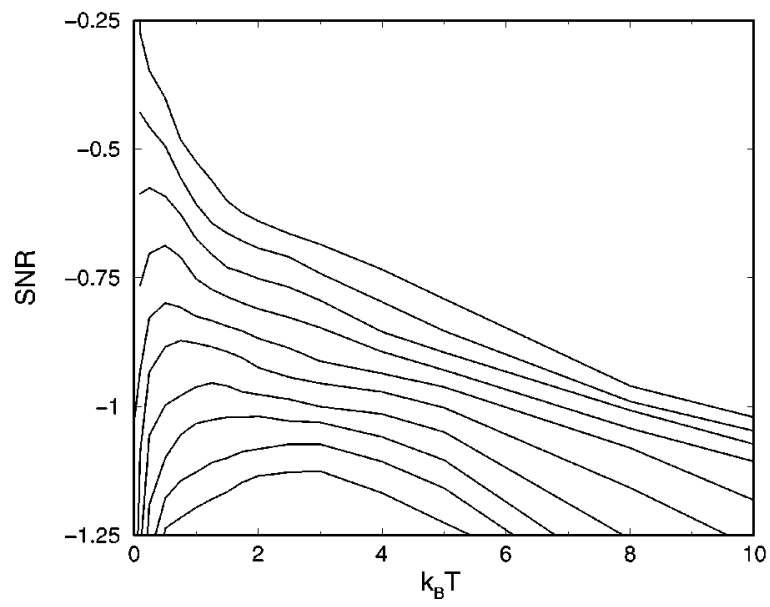

The signal at the first few sites is monotonically weakened as a function of the temperature (as it is at all sites in the harmonic chain), but the SNR is enhanced with increasing temperature for sites farther away until it reaches a maximum (thermal resonance); beyond that it decreases. Note that the optimal temperature increases with distance from the first site. For the particular parameters used in this simulation the thermal resonance occurs most clearly at temperatures in the range $k_{B} T<4$ for sites between the 8 th and the 15 th; these details can of course be modified by changing the parameters (see Sec. V).

Complementary results for SNR curves as a function of the site for different temperatures are shown in the second panel in Fig. 5. The resonance effects are evidenced by the crossings of the different curves. The crossings reflect the rise and subsequent drop in the SNR at a given site, and the fact that the curves cross at different sites confirms that the thermal resonance temperature varies from site to site.

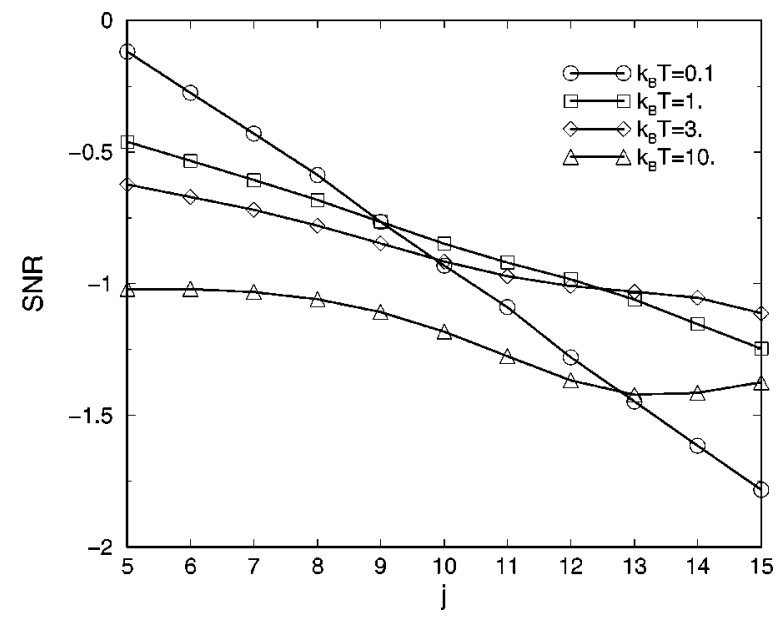

FIG. 5. First panel: SNR curves for different sites (from $j=6$ to 15, top to bottom) along the anharmonic chain as a function of the temperature for the standard case: $A=0.5, k^{\prime}=5, \gamma=0.2, \omega_{0}=1$. The optimal temperature increases with distance from the first site. Second panel: SNR as a function of the site for different temperatures. 
The nonmonotonic behavior of the SNR with temperature is due to the fact that while the thermal power increases with $T$, the signal power at first also increases, and more rapidly than the thermal power. This is exactly the condition for the existence of stochastic resonance formulated by Dykman et al. $[26,27,33]$ for single potentials, but that theory has not yet been generalized to extended systems. The fast increase of the signal power is consistent with trends found earlier for transmission of pulses along discrete arrays [13]. We found that a pulse in a hard anharmonic chain travels more rapidly with increasing temperature (except for the lowest temperatures, see below), while in a harmonic array a change in temperature has no effect on pulse speed. While a pulse broadens with increasing temperature in both arrays, the pulse in the anharmonic array remains relatively more compact. At sufficiently high temperatures the signal power at a given site becomes independent of $T$ (as in the harmonic chain) because the signal response has reached its maximum value; a further increase in temperature only affects the signal further down the chain. The "crossover" temperature of the signal power from the increasing to the saturated behavior depends on the chain and signal parameters, a dependence explored in the next section.

The fact that the SNR for the sites shown is very low (essentially zero) for the lowest temperatures at sites beyond the first few shows that for a purely anharmonic chain the signal essentially stops beyond the first few sites, whereas in the harmonic example the signal reaches all the sites shown even at the lowest temperatures shown. This is also consistent with the behavior shown earlier for the transmission of a pulse [13]: the pulse velocity at sufficiently low temperatures is actually lower in the hard chain than in the harmonic case. Had we included a harmonic potential contribution equal to that of the harmonic chain, the SNR vs $k_{B} T$ curves would start at the same values as in Fig. 4, but for sufficiently distant sites from the first they would still be nonmonotonic. We have omitted a harmonic contribution to present the thermal resonance effect in its purest form.

The apparently monotonic behavior of the first few sites is due to the fact that for the temperatures shown the signal reaches these sites in any case. A resonance at these sites would be seen for different parameter values and/or at even lower temperatures.

Our preceding descriptions point to another interesting measure of a thermal resonance, namely, the propagation length $\Lambda$, defined as the number of sites (i.e., distance along the chain) for which the SNR exceeds a certain threshold value. Figure 6 presents the temperature dependence of $\Lambda$ for harmonic and anharmonic arrays and an arbitrarily chosen $\mathcal{R}$-threshold value of -1.2 . Since the harmonic array does not exhibit thermal resonance, a monotonic decay of $\Lambda$ with increasing temperature is observed. On the other hand, the hard chain shows a maximum for a moderate temperature. Again, the particular values of optimal temperature $\left(k_{B} T\right.$ $\approx 3)$ and optimal distance $(\Lambda \approx 17)$ can be modified by choosing different parameters and/or different SNR thresholds, but the qualitative behavior persists as seen in Fig. 6 .

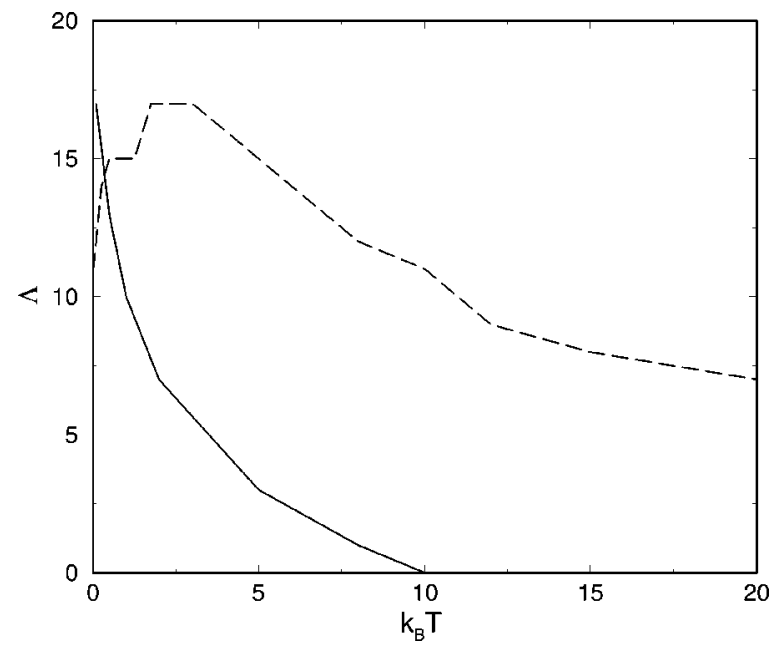

FIG. 6. Propagation length $\Lambda$ as a function of the temperature for the anharmonic chain (standard case) and harmonic chain with $k=1$ (other parameters as in the standard anharmonic case). The threshold value is $\mathcal{R}=-1.2$.

\section{PARAMETER DEPENDENCES}

The parameters that can be varied in our model are the amplitude $A$ and frequency $\omega_{0}$ of the velocity of the first site, the coupling parameter $k^{\prime}$, and the damping coefficient $\gamma$.

The first panel of Fig. 7 shows the SNR curves as a function of temperature for a lower amplitude than in the standard case. Comparison with the first panel of Fig. 5 shows that the overall SNR is now (of course) lower and that the resonance temperature at each site has increased. The latter behavior indicates that for lower amplitudes the crossover temperature from an increasing signal to a saturated signal increases as the signal weakens. The second panel shows results for weaker coupling. Again the overall SNR is lower and the resonance temperature higher at each site. A weaker coupling thus has the effect of weakening the signal.

The first panel of Fig. 8 shows the SNR curves as a function of temperature for higher damping. Comparison with the first panel of Fig. 5 shows that the overall SNR is again lower than in the standard case and the resonance temperature higher. The same trends are observed with a higher driving frequency as shown in the second panel of Fig. 8. Again, each of these changes leads to an effectively weakened signal.

Figures 7 and 8 illustrate clear trends. Decreasing $A$ or $k^{\prime}$, or increasing $\gamma$ or $\omega_{0}$, lead to the following consequences: (a) the SNR at any given site and temperature decreases; (b) the SNR resonance temperature increases at any given site; and (c) at a given temperature the resonance occurs at a site closer to the first. These trends are consistent with those found earlier for transmission of pulses along similar arrays [13]. We found that a pulse in a hard anharmonic chain travels more rapidly and relatively more compactly with increasing amplitude, decreasing damping, increasing coupling, or increasing driving frequency. By contrast, in a harmonic array changes in temperature or in the signal and damping parameters have no effect on pulse speed. 

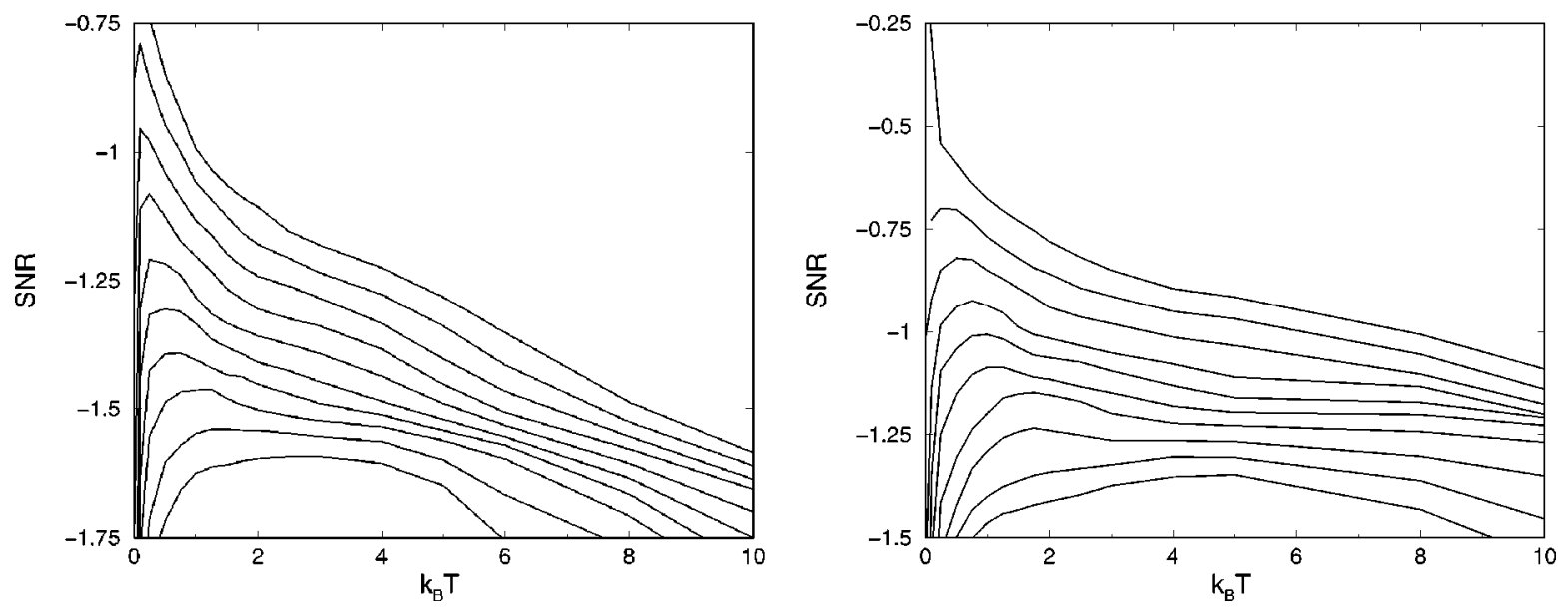

FIG. 7. First panel: SNR curves for different sites (from $j=4$ to 13 , top to bottom) along the anharmonic chain as a function of the temperature for the lower-amplitude case: $A=0.25, k^{\prime}=5, \gamma=0.2, \omega_{0}=1$. Second panel: SNR curves for the weaker-coupling case: $A$ $=0.5, k^{\prime}=3, \gamma=0.2, \omega_{0}=1$.

\section{CONCLUSION}

We have demonstrated thermal resonances in simple onedimensional arrays of masses connected by hard springs. Our chains are not overdamped and hence include inertial effects. We have shown that the distance and amplitude of propagation of a signal imposed at one end of the chain can each be optimized by tuning the temperature of the system. The resonance behavior reflects the temperature dependence of the distance traveled by the front of a signal and the dispersion of this front once steady state has been reached. At a fixed temperature, any parameter change that leads to an increase in velocity of propagation tends to increase the range of sites where the signal can be detected as well as the SNR at a given site. Increased velocity of propagation is also associated with a lowering of the resonance temperature for a given site.

A complete understanding of the behavior of these simple anharmonic arrays in a thermal environment requires and relies on a number of other inquiries, some of which we have undertaken. One concerns the distribution of energy, and the persistence and mobility of energy fluctuations, in thermal equilibrium $[12,14]$. We have found that soft anharmonic chains (that is, chains with soft interaction potentials) experience greater energy fluctuations than harmonic chains, which in turn experience greater fluctuations than hard chains. This is a direct consequence of the virial theorem. We have also established that fluctuations are mobile in harmonic and hard chains but not in the soft. Most interestingly, thermal fluctuations travel most rapidly and remain localized over considerably greater distances in the hard chain. These results in turn can lead to very different transition rate statistics and effective damping coefficients for a bistable impurity embedded in each of these arrays at a given temperature [14].

Another interesting line of inquiry concerns the propagation of an energy pulse along such arrays. As mentioned earlier, we have found that the propagation of an energy pulse in a hard anharmonic array can be enhanced by immersing the array in a thermal bath, and that hard anharmonicity in the springs causes a tight and persistent packing of the energy [13].

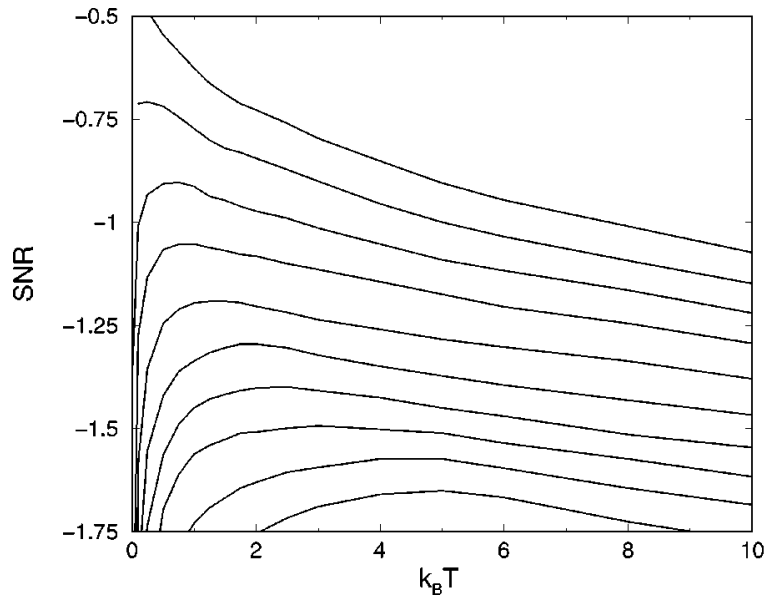

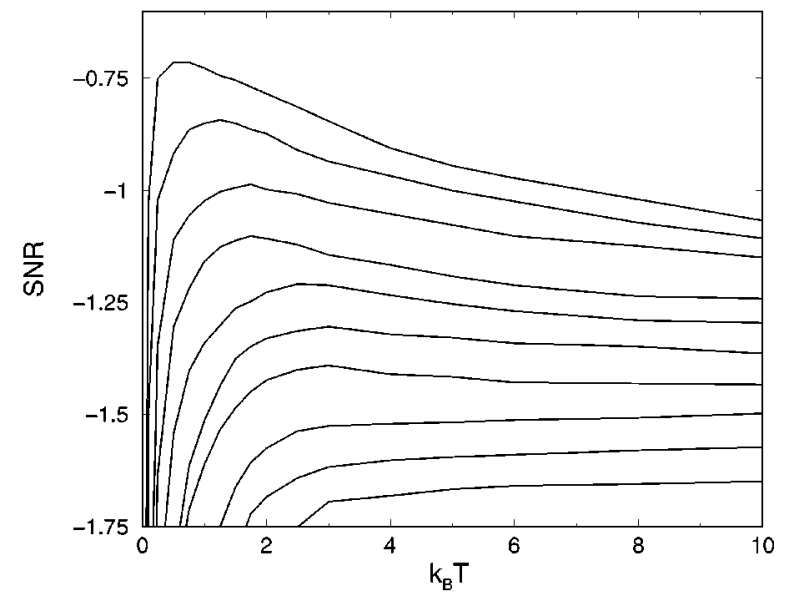

FIG. 8. SNR curves for sites 4 to 13 (top to bottom) for the higher damping case: $A=0.5, k^{\prime}=5, \gamma=0.5$, $\omega_{0}=1$. Second panel: SNR curves for the higher frequency case: $A=0.5, k^{\prime}=5, \gamma=0.2, \omega_{0}=2$. 
Almost all of our results are numerical. Analytic results for the harmonic array provide some insights and point toward the possibility that an approach based on linearization methods [34] together with a linear response theory [33] might lead to analytic insights for nonlinear arrays. We are currently exploring these possibilities.

The thermal resonances and other interesting behavior that we have found for simple hard anharmonic chains are likely to be prototypical and therefore applicable to many other discrete systems with anharmonic interactions. While many other systems have been investigated in which a careful balance of parameters at zero temperature or a manipulation of external noise lead to interesting localization, resonance, and synchronization phenomena, our systems are among the simplest generic systems in which the temperature can be used as the tuning parameter to achieve similar effects.

\section{ACKNOWLEDGMENTS}

R.R. gratefully acknowledges the support by the Ministerio de Educación y Cultura through Postdoctoral Grant No. PF-98-46573147. A.S. acknowledges sabbatical support from DGAPA-UNAM. This material is based upon work supported in part by a grant from the University of California Institute for Mexico and the United States (UC MEXUS) and the Consejo Nacional de Ciencia y Tecnologia de Mexico (CONACYT). This work was supported in part by the Engineering Research Program of the Office of Basic Energy Sciences at the U.S. Department of Energy under Grant No. DE-FG03-86ER13606.

\section{APPENDIX: HARMONIC CHAIN}

In this appendix we consider a harmonic chain and calculate analytically some of the numerical results presented or invoked in the body of the paper. Although the behavior of linear chains is in general well understood, it is useful to present results in the particular context of this work. These are not readily available in the literature.

\section{Zero temperature, with signal}

Consider first an infinite linear chain $(-\infty<n<\infty)$ at zero temperature, that is, in the absence of fluctuations (but with the dissipative contribution). The equations of motion then are

$$
\ddot{x}_{n}=k\left(x_{n+1}+x_{n-1}-2 x_{n}\right)-\gamma \dot{x}_{n},
$$

with the signal $\dot{x}_{0}=A \sin \left(\omega_{0} t\right)$ applied at site $n=0$. We conjecture the quasistationary solution

$$
x_{n}^{s}(t)=-\frac{A}{\omega_{0}} e^{-|n| \mu} \cos \left(\omega_{0} t+|n| b\right)+c_{n},
$$

where the superscript $s$ denotes the presence of the signal. The constants $\mu$ and $b$ are to be determined. The additive constant $c_{n}$ must be independent of $n$-it simply represents an overall translation of the chain (because it is not an- chored) but otherwise does not contribute to the velocity analysis. The velocities associated with Eq. (A2) are

$$
\dot{x}_{n}^{s}(t)=A e^{-|n| \mu} \sin \left(\omega_{0} t+|n| b\right) .
$$

This result is clearly consistent with the imposed signal at $n=0$. The solution (A3) can be used to calculate the zerotemperature signal according to Eq. (5),

$$
S_{n}^{S}\left(\omega_{0}\right)=\int_{-\infty}^{+\infty} e^{-i \omega_{0} \tau}\left\langle\dot{x}_{n}(t) \dot{x}_{n}(t+\tau)\right\rangle d \tau .
$$

The average (which in this deterministic case is only over time) eliminates rapidly oscillating contributions, leaving only a $\delta$-function type of contribution. The result is the power spectral density

$$
S_{n}^{S}\left(\omega_{0}\right)=\frac{A^{2}}{2} e^{-2|n| \mu} .
$$

To find the constant $\mu$ we substitute Eq. (A2) into Eq. (A1) and set the coefficients of $e^{i \omega_{0} t}$ and those of $e^{-i \omega_{0} t}$ equal to zero (one resulting equation is simply the complex conjugate of the other). This immediately leads to the relation

$$
-\omega_{0}^{2}=k\left(e^{-\mu+i b}+e^{\mu-i b}-2\right)-i \gamma \omega_{0} .
$$

We make the substitution $u \equiv e^{-\mu+i b}$ and note the symmetry in $u$ and $1 / u$,

$$
k\left(u+\frac{1}{u}-2\right)+\omega_{0}^{2}-i \gamma \omega_{0}=0 .
$$

Next we multiply through by $u$ and solve the resulting quadratic equation,

$$
u_{ \pm}=\frac{-\left(\omega_{0}^{2}-2 k-i \gamma \omega_{0}\right) \pm \sqrt{\left(\omega_{0}^{2}-2 k-i \gamma \omega_{0}\right)^{2}-4 k^{2}}}{2 k} .
$$

Note that $u_{+} u_{-}=1$, so if one solution is $e^{-\mu+i b}$, then the other is $e^{\mu-i b}$. Which is which is not clear at this point. $\mu$ must be positive for a physically acceptable solution and this will be used below to sort out the choice.

To extract $\mu$ we calculate the ratio $u_{+} / u_{-}^{*}$, which is either $e^{2 \mu}$ or $e^{-2 \mu}$. If $u_{+}=e^{-\mu+i b}$ then $u_{+} / u_{-}=e^{-2 \mu}$. If, on the other hand, $u_{+}=e^{\mu-i b}$ then $u_{+} / u_{-}=e^{2 \mu}$. Which it is will be seen at the end when we inspect the magnitude of the result.

In calculating the ratio $u_{+} / u_{-}^{*}$ one must exercise caution in taking complex conjugates because of the square root in Eq. (A8); it is not appropriate to simply change every $i$ to a $-i$ in such an expression if one does not know the signs of the terms inside the square root. It is helpful to write each complex number in terms of an amplitude and a phase,

$$
\left(\omega_{0}^{2}-2 k-i \gamma \omega_{0}\right) \equiv 2 k \alpha e^{i \beta},
$$

so that 


$$
\begin{gathered}
2 k \alpha=\left[\left(\omega_{0}^{2}-2 k\right)^{2}+\gamma^{2} \omega_{0}^{2}\right]^{1 / 2}, \\
\beta=\tan ^{-1}\left(\frac{-\gamma \omega_{0}}{\omega_{0}^{2}-2 k}\right) .
\end{gathered}
$$

Similarly, with

$$
\left(\omega_{0}^{2}-2 k-i \gamma \omega_{0}\right)^{2}-4 k^{2} \equiv 4 k^{2} \nu^{2} e^{2 i \epsilon},
$$

we find

$$
\begin{gathered}
4 k^{2} \nu^{2}=\omega_{0}\left[\omega_{0}^{2}\left(\omega_{0}^{2}-4 k-\gamma^{2}\right)^{2}+4 \gamma^{2}\left(\omega_{0}^{2}-2 k\right)^{2}\right]^{1 / 2} \\
2 \epsilon=\tan ^{-1} \frac{-2 \gamma\left(\omega_{0}^{2}-2 k\right)}{\omega_{0}\left(\omega_{0}^{2}-4 k-\gamma^{2}\right)}
\end{gathered}
$$

Then

$$
u_{+}=-\alpha e^{i \beta}+\nu e^{i \epsilon}, \quad u_{-}=-\alpha e^{i \beta}-\nu e^{i \epsilon},
$$

and the ratio $u_{+} / u_{-}^{*}$ is thus real. Multiplying top and bottom of this ratio by $u_{-}$gives

$$
\frac{u_{+}}{u_{-}^{*}}=\frac{u_{+} u_{-}}{u_{-}^{*} u_{-}}=\frac{1}{\left|u_{-}\right|^{2}} .
$$

Further and explicitly

$$
\begin{aligned}
\left|u_{-}\right|^{2} & =\left(-\alpha e^{i \beta}-\nu e^{i \epsilon}\right) \times\left(-\alpha e^{-i \beta}-\nu e^{-i \epsilon}\right) \\
& =\alpha^{2}+\nu^{2}+2 \alpha \nu \cos (\beta-\epsilon) .
\end{aligned}
$$

All that remains is the evaluation of the resulting expressions. In summary

$$
\alpha^{2}+\nu^{2}+2 \alpha \nu \cos (\beta-\epsilon)=e^{-2 \mu},
$$

or

$$
\alpha^{2}+\nu^{2}+2 \alpha \nu \cos (\beta-\epsilon)=e^{+2 \mu} .
$$

The choice is determined by whether the result is $<1$ (in which case the first equality holds) or $>1$ (in which case it is the second). The quantities $\alpha, \nu, \beta$, and $\epsilon$ are thus completely defined in terms of the system parameters.

We have tested our numerical simulations against the prediction (A5) for large ranges of parameter values and have found agreement to five significant figures.

The solutions presented in this section do not obey $\mathrm{N}$-periodic boundary conditions. It is fairly straightforward using an imagelike method to construct explicitly periodic solution by applying the signal at $0, \pm N, \pm 2 N, \ldots$ but the changes would be exponentially small in $N$ and unimportant for sufficiently long chains. The quasistationary behavior assumed above for sites not too distant from $n=0$ sets in long before the signal at $n=0$ reaches sites $\pm N$.

\section{Finite temperature, no signal}

Now we solve the equations of motion

$$
\ddot{x}_{n}=k\left(x_{n+1}+x_{n-1}-2 x_{n}\right)-\gamma \dot{x}_{n}+f_{n}(t)
$$

without a signal. We are again interested in the stationary behavior.

We define the Fourier transform and its inverse,

$$
y_{q}=\sum_{n=0}^{N-1} x_{n} e^{2 \pi i q n}, \quad x_{n}=\frac{1}{N} \sum_{q=0}^{N-1} y_{q} e^{-2 \pi i n q} .
$$

Transforming Eq. (A18) immediately leads to

$$
\ddot{y}_{q}+4 k \sin ^{2}\left(\frac{\pi q}{N}\right) y_{q}+\gamma \dot{y}_{q}=F_{q}(t),
$$

where the inhomogeneous term is the transform of the noise,

$$
F_{q}(t) \equiv \sum_{n=0}^{N-1} f_{n}(t) e^{2 \pi i q n}
$$

The solution of this second order inhomogeneous differential equation is of standard form. The initial conditions (which we will take to be zero) are unimportant since we seek the long-time behavior,

$$
\begin{aligned}
y_{q}^{0}(t)= & \lim _{t \rightarrow \infty} \frac{1}{\left[r_{2}(q)-r_{1}(q)\right]} \int_{0}^{t} F_{q}(\tau)\left[e^{r_{2}(q)(t-\tau)}\right. \\
& \left.-e^{r_{1}(q)(t-\tau)}\right] d \tau
\end{aligned}
$$

and consequently

$$
\begin{aligned}
\dot{y}_{q}^{0}(t)= & \lim _{t \rightarrow \infty} \frac{1}{\left[r_{2}(q)-r_{1}(q)\right]} \int_{0}^{t} F_{q}(\tau)\left[r_{2}(q) e^{r_{2}(q)(t-\tau)}\right. \\
& \left.-r_{1}(q) e^{r_{1}(q)(t-\tau)}\right] d \tau
\end{aligned}
$$

where

$$
r_{1,2}(q)=-\frac{\gamma}{2} \pm \sqrt{\left(\frac{\gamma}{2}\right)^{2}-4 k \sin ^{2}\left(\frac{\pi q}{N}\right)} .
$$

The superscript 0 is used to stress the absence of a signal.

With this result and the correlation function for the thermal fluctuations that follow immediately from Eq. (3),

$$
\left\langle F_{q}\left(t_{1}\right) F_{q^{\prime}}\left(t_{2}\right)\right\rangle=2 \gamma k_{B} T \delta\left(t_{1}-t_{2}\right) N \delta_{q,-q^{\prime}},
$$

we obtain upon integration and Fourier inversion the velocity correlation function

$$
\begin{aligned}
C_{n}^{0}(\tau) \equiv & \left\langle\dot{x}_{n}(t) \dot{x}_{n}(t+\tau)\right\rangle \\
= & \frac{\gamma k_{B} T}{N} \sum_{q=0}^{N-1} \frac{1}{\left[r_{2}^{2}(q)-r_{1}^{2}(q)\right]}\left[r_{1}(q) e^{r_{1}(q) t}\right. \\
& \left.-r_{2}(q) e^{r_{2}(q) t}\right] .
\end{aligned}
$$




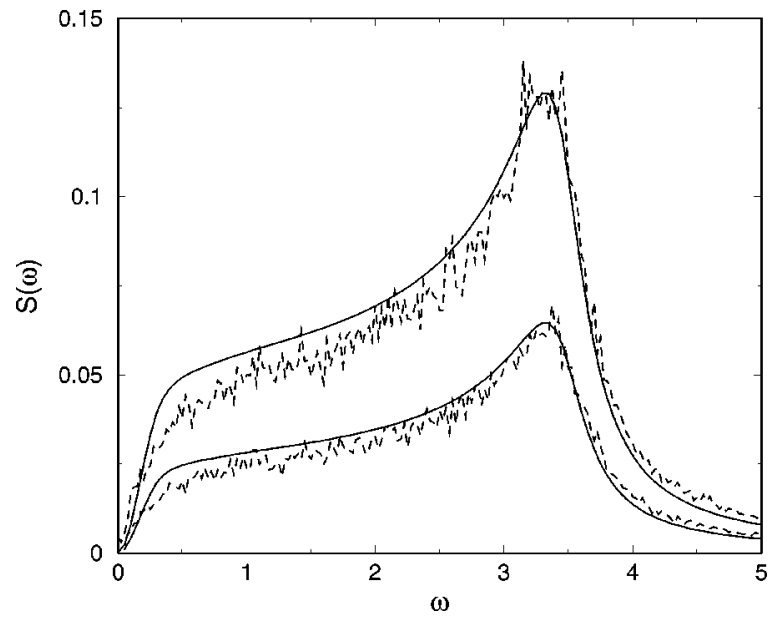

FIG. 9. Spectrum $S_{n}^{0}(\omega)$ for any site at two temperatures, $k_{B} T$ $=0.05$ (lower curves) and $k_{B} T=0.1$ (upper curves). The smooth curves are the predictions in Eq. (A27) and the jagged curves are the numerical simulation results averaged over 500 runs. The irregularities can be eliminated at great CPU time cost by refining the time and/or frequency meshes and/or increasing the number of runs. Other parameters: $k=3, \gamma=0.5$.

We note in passing that this reduces to the standard correlation function for a single harmonic oscillator in a heat bath, $C_{n}^{0}(\tau) \rightarrow k_{B} T e^{-\gamma \tau}$, when the coupling coefficient $k \rightarrow 0$. The associated power spectral density is

$$
\begin{aligned}
S_{n}^{0}(\omega) & =2 \int_{0}^{\infty} d \tau C_{n}^{0}(\tau) \cos (\omega \tau) \\
& =\frac{2 \gamma k_{B} T \omega^{2}}{N} \sum_{q=0}^{N-1} \frac{1}{\left[r_{1}^{2}(q)+\omega^{2}\right]\left[r_{2}^{2}(q)+\omega^{2}\right]} .
\end{aligned}
$$

Figure 9 shows the predicted spectrum (smooth curves)

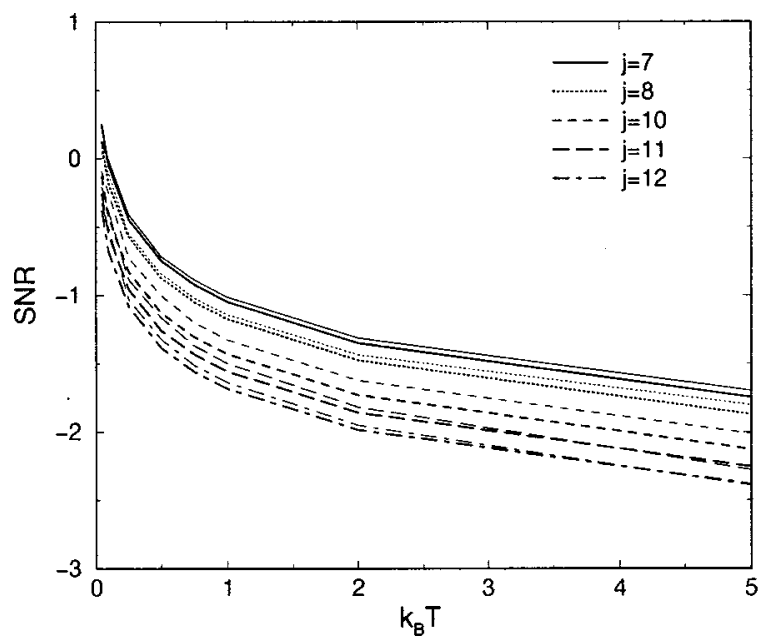

and the corresponding simulation results for two different temperatures. The agreement is typical of broad parameter ranges.

\section{Finite temperature and signal: SNR}

Finally, we deal with the full equation of motion

$$
\ddot{x}_{n}=k\left(x_{n+1}+x_{n-1}-2 x_{n}\right)-\gamma \dot{x}_{n}+f_{n}(t)
$$

with the signal $\dot{x}_{0}=A \sin \left(\omega_{0} t\right)$ applied at site $n=0$. The fluctuation $f_{0}$ is set to zero since the velocity of that site is fixed by the signal.

The Fourier transform is defined as before, but in transforming the equations of motion we must take into account that Eq. (A28) does not hold for $n=0$. We thus multiply Eq. (A28) by $e^{2 \pi i q n}$ and sum over $n$ but only from $n=1$ to $N$ -1 ,

$$
\sum_{n=1}^{N-1} x_{n} e^{2 \pi i q n}=y_{q}-x_{0} .
$$

Proceeding in this manner we obtain in place of Eq. (A20)

$$
\ddot{y}_{q}+4 k \sin ^{2}\left(\frac{\pi q}{N}\right) y_{q}+\gamma \dot{y}_{q}=U_{q}(t)
$$

where the inhomogeneous term now is

$$
U_{q}(t)=F_{q}(t)+k\left(2 x_{0}-x_{1}-x_{N-1}\right)+\gamma \dot{x}_{0}+\ddot{x}_{0} .
$$

The terms containing $x_{0}$ and its derivatives are known; $x_{1}$ and $x_{N-1}$ (they are equal by symmetry) would have to be found "self-consistently" ex-post (see below).

Equation (A30) is again a linear inhomogeneous differential equation with constant coefficients. Its solution has two additive contributions,

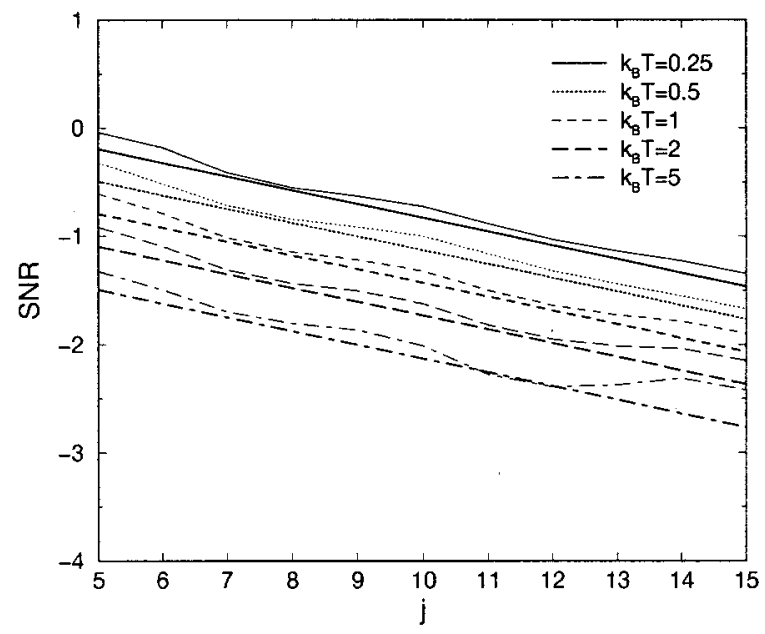

FIG. 10. First panel: Typical SNR curves as a function of temperature for sites 7, 8, 10, 11, and 12 along a harmonic chain with $k$ $=3, \gamma=0.5, \omega_{0}=1.0$, and $A=0.5$. The numerical simulation results (thin lines) in each case lie above the analytic curves (thick lines). Second panel: SNR as a function of site for different temperatures. 


$$
y_{q}(t)=y_{q}^{h o m}(t)+y_{q}^{i n h}(t) .
$$

The portion $y_{q}^{\text {hom }}(t)$ is the solution of the homogeneous equation, that is, with $U_{q}(t)=0$, and the contribution $y_{q}^{i n h}(t)$ is due to the inhomogeneity.

Suppose that $F_{q}(t)$ were equal to zero. The solution in this case, which we denote by $y_{q}^{s}(t)$ is exactly the solution of Sec. A1; that is, the inverse transform of

$$
y_{q}^{s}(t)=y_{q}^{s, h o m}(t)+y_{q}^{s, i n h}(t)
$$

at long times must be precisely Eq. (A2). Now consider the consequences of again including $F_{q}(t)$. The homogeneous part of the solution has to be exactly as before, i.e., $y_{q}^{\text {hom }}$ $=y_{q}^{s, h o m}$ because the homogeneous part of the equation has not changed, the constraint at $x_{0}$ also has not changed, and initial conditions are in any case immaterial at long times. However, the inhomogeneous part of the solution does change, and it does so in two ways. First and most important is the direct contribution of the thermal fluctuations to the solution. Second, and less important, is the change in $x_{1}$ and $x_{-1}$ (which is the same as that of $x_{1}$ ) caused by the addition of the noise, and the change that this in turn imposes on the other displacements. This contribution is more difficult to calculate and affects only sites near $n=0$. Ignoring the latter we thus assume the solution

$$
y_{q}(t)=y_{q}^{s}(t)+y_{q}^{0}(t)
$$

where $y_{q}^{s}(t)$ is given in Eq. (A33) and $y_{q}^{0}(t)$ in Eq. (A22).

The correlation function and spectrum are then simply the sums of the correlation functions and spectra for the system with the signal but no fluctuations (zero temperature) and those for the thermalized system in the absence of the signal. Thus, the spectrum is the sum of Eq. (A5) (appropriately weighted by a delta function that places it at $\omega_{0}$ ) and Eq. (A27). In particular, the SNR for the harmonic system then finally is

$$
\begin{aligned}
\mathcal{R}_{n}\left(\omega_{0}\right) & =\log _{10}\left(\frac{\frac{A^{2}}{2} e^{-2 n \mu}}{\frac{2 \gamma k_{B} T \omega^{2}}{N} \sum_{q=0}^{N-1} \frac{1}{\left[r_{1}^{2}(q)+\omega^{2}\right]\left[r_{2}^{2}(q)+\omega^{2}\right]}}\right),
\end{aligned}
$$

which decreases monotonically with $T$ as well as with $n$. This result is consistent with the linear response theory approach introduced and widely applied by Dykman et al. [33]. Comparisons of this analytic result with numerical simulations are shown in Fig. 10 (cf. Fig. 4). Equation (A35) clearly captures the correct behavior; the small discrepancies between analytic and numerical results (which always lie above the analytic curves) reflect the omitted terms discussed above.
[1] R. Benzi, A. Sutera, and A. Vulpiani, J. Phys. A 18, 2239 (1985).

[2] K. Wiesenfeld and F. Moss, Nature (London) 373, 33 (1995); A.R. Bulsara and L. Gammaitoni, Phys. Today 49 (3), 39 (1996); D.G. Luchinsky, P.V.E. McClintock, and M.I. Dykman, Rep. Prog. Phys. 61, 889 (1998); Stochastic Dynamics, edited by L. Schimansky-Geier and T. Poeschel (SpringerVerlag, Berlin, 1997); L. Gammaitoni, P. Hänggi, P. Jung, and F. Marchesoni, Rev. Mod. Phys. 70, 223 (1998); the focus issue Chaos 7, 1997 features a number of papers that review recent work on stochastic resonance.

[3] J.F. Lindner, S. Chandramouli, A.R. Bulsara, M. Löcher, and W.L. Ditto, Phys. Rev. Lett. 81, 5048 (1998).

[4] M. Löcher, D. Cigna, and E.R. Hunt, Phys. Rev. Lett. 80, 5212 (1998); J.F. Lindner, B.K. Meadows, W.L. Ditto, M.E. Inchiosa, and A.R. Bulsara, ibid. 75, 3 (1995); J.F. Lindner, B.K. Meadows, W.L. Ditto, M.E. Inchiosa, and A.R. Bulsara, Phys. Rev. E 53, 2081 (1996); Y. Zhang, G. Hu, and L. Gammaitoni, ibid. 58, 2952 (1998).

[5] M. Löcher, D. Cigna, E.R. Hunt, G.A. Johnson, F. Marchesoni, L. Gammaitoni, M.E. Inchiosa, and A.R. Bulsara, Chaos 8, 604 (1998); G. Giacomelli, F. Marin, and I. Rabbiosi, Phys. Rev. Lett. 82, 675 (1999); D. Dingklage, C. Wilke, and T. Klinger, Phys. Plasmas 6, 2968 (1999); A.D. Hibbs and B.R. Whitecotton, in Applied Nonlinear Dynamics and Stochastic Systems Near the Millennium, edited by James B.
Kadte and Adi Bulsara, AIP Conf. Proc. 411 (AIP, New York, 1997), p. 227.

[6] D.S. Leonard and L.E. Reichl, Phys. Rev. E 49, 1734 (1994).

[7] S. Kádár, J. Wang, and K. Showalter, Nature (London) 391, 770 (1998)

[8] P. Jung, et al., Chaos 8, 567 (1998).

[9] H. Zhonghuai, L. Yang, and H. Xin, J. Chem. Phys. 111, 1592 (1999); L. Yang, Z. Hou, and H. Xin, ibid. 110, 3591 (1999).

[10] L. Stode and P.I. Saparin, in Applied Nonlinear Dynamics and Stochastic Systems Near the Millennium (Ref. [5]), p. 341; B. Wand, A. Barcilon, and Z. Fang, J. Atmos. Sci. 56, 5 (1999).

[11] E. Simonotto et al., Phys. Rev. Lett. 78, 1186 (1997); F.Y. Chiou-Tan et al., Int. J. Bifurcation Chaos Appl. Sci. Eng. 6, 1389 (1996); S. Blesic, S. Milosevic, D. Stratimirovic, and M. Ljubisavljevic, Physica A 268, 275 (1999); R. Srebro and P. Malladi, Phys. Rev. E 59, 2566 (1999); A.B. Uzdensky, Electro- Magnetobiol. 18, 67 (1999).

[12] R. Reigada, A.H. Romero, A. Sarmiento, and K. Lindenberg, J. Chem. Phys. 111, 1373 (1999).

[13] A. Sarmiento, R. Reigada, A.H. Romero, and K. Lindenberg, Phys. Rev. E 60, 5317 (1999).

[14] R. Reigada, A. Sarmiento, A.H. Romero, J.M. Sancho, and K. Lindenberg, J. Chem. Phys. 112, 10615 (2000).

[15] J. Garcia-Ojalvo and J. M. Sancho, Noise in Spatially Extended Systems (Springer, New York, 1999).

[16] M. Löcher, N. Chatterjee, F. Marchesoni, W.L. Ditto, and E.R. Hunt, e-print cond-mat/0003048. 
[17] P. Jung and G. Mayer-Kress, Phys. Rev. Lett. 74, 2130 (1995).

[18] F. Castelpoggi and H. Wio, Phys. Rev. E 57, 5112 (1998).

[19] J.P. Keener, Chaos 8, 175 (1998).

[20] W.C. Cole, J.B. Picone, and N. Sperelakis, Biophys. J. 53, 809 (1988).

[21] S. Flach and C.R. Willis, Phys. Rep. 295, 181 (1998).

[22] C. Laroche, T. Dauxois, and M. Peyrard, Am. J. Phys. 68, 552 (2000).

[23] T. Prosen and D.K. Campbell, e-print chao-dyn/9908021.

[24] P.J. Martinez, L.M. Floria, F. Falo, and J.J. Mazo, Europhys. Lett. 45, 444 (1999).

[25] C. Giardina, R. Livi, A. Politi, and M. Vassalli, Phys. Rev. Lett. 84, 2144 (2000).

[26] M.I. Dykman, D.G. Luchinsky, R. Mannella, P.V.E. McClintock, N.D. Stein, and N.G. Stocks, J. Stat. Phys. 70, 479 (1993).

[27] N.G. Stocks, N.D. Stein, and P.V.E. McClintock, J. Phys. A 26, L385 (1993).

[28] I.Kh. Kaufman, D.G. Luchinsky, P.V.E. McClintock, S.M.
Soskin, and N.D. Stein, Phys. Lett. A 220, 219 (1996).

[29] A.N. Grigorenko, S.I. Nikitin, and G.V. Roschepkin, Phys. Rev. E 56, R4907 (1997).

[30] T. C. Gard, Introduction to Stochastic Differential Equations, Monographs and Textbooks in Pure and Applied Mathematics Vol. 114 (Marcel Dekker, New York, 1987).

[31] R. Toral, in Computational Field Theory and Pattern Formation, Lecture Notes in Physics Vol. 448 (Springer-Verlag, Berlin, 1995).

[32] D.W. Brown, L.J. Bernstein, and K. Lindenberg, Phys. Rev. E 54, 3352 (1996).

[33] M.I. Dykman, R. Mannella, P.V.E. McClintock, and N.G. Stocks, Phys. Rev. Lett. 65, 2606 (1990); Pis'ma Zh. Éksp. Teor. Fiz. 52, 180 (1990) [JETP Lett. 52, 141 (1990)]; Phys. Rev. Lett. 68, 2985 (1992); M.I. Dykman, D.G. Luchinsky, R. Mannella, P.V.E. McClintock, N.D. Stein, and N.G. Stocks, J. Stat. Phys. 70, 462 (1993).

[34] A. Bulsara, K. Lindenberg, and K.E. Shuler, J. Stat. Phys. 27, 787 (1982). 